\title{
Large Proteoglycan Complexes and Disturbed Collagen Architecture in the Corneal Extracellular Matrix of Mucopolysaccharidosis Type VII (Sly Syndrome)
}

\author{
Robert D. Young, ${ }^{1}$ Petra Liskova, ${ }^{2}$ Christian Pinali, ${ }^{1}$ Barbara P. Palka, ${ }^{1}$ Michalis Palos, ${ }^{3}$ \\ Katerina Jirsova, ${ }^{2}$ Enkela Hrdlickova, ${ }^{3}$ Marketa Tesarova, ${ }^{4}$ Milan Elleder, ${ }^{5}$ Jiri Zeman, ${ }^{4}$ \\ Keith M. Meek, ${ }^{1}$ Carlo Knupp, ${ }^{1}$ and Andrew J. Quantock ${ }^{1}$
}

Purpose. Deficiencies in enzymes involved in proteoglycan (PG) turnover underlie a number of rare mucopolysaccharidoses (MPS), investigations of which can considerably aid understanding of the roles of PGs in corneal matrix biology. Here, the authors analyze novel pathologic changes in MPS VII (Sly syndrome) to determine the nature of PG-collagen associations in stromal ultrastructure.

Methods. Transmission electron microscopy and electron tomography were used to investigate PG-collagen architectures and interactions in a cornea obtained at keratoplasty from a 22-year-old man with MPS VII, which was caused by a compound heterozygous mutation in the GUSB gene.

Results. Transmission electron microscopy showed atypical morphology of the epithelial basement membrane and Bowman's layer in MPS VII. Keratocytes were packed with cytoplasmic vacuoles containing abnormal glycosaminoglycan (GAG) material, and collagen fibrils were thinner than in normal cornea and varied considerably throughout anterior (14-32 nm), mid (13-42 nm), and posterior (17-39 nm) regions of the MPS VII stroma. PGs viewed in three dimensions were striking in appearance in that they were significantly larger than PGs in normal cornea and formed highly extended linkages with multiple collagen fibrils.

From the ${ }^{1}$ Structural Biophysics Group, School of Optometry and Vision Sciences, Cardiff University, Cardiff, Wales, United Kingdom; the ${ }^{2}$ Ocular Tissue Bank and Laboratory of the Biology and Pathology of the Eye, Institute of Inherited Metabolic Disorders, the Departments of ${ }^{3}$ Ophthalmology and ${ }^{4}$ Pediatrics and Adolescent Medicine, and the ${ }^{5}$ Institute of Inherited Metabolic Disorders, First Faculty of Medicine, Charles University in Prague and General University Hospital in Prague, Prague, Czech Republic.

Supported by Biotechnology and Biological Sciences Research Council UK Project Grant BB/F022077/1 (CK, AJQ), Engineering and Physical Sciences Research Council UK Research Grant EP/F034970/1 (AJQ, KMM, RDY), and Medical Research Council UK Programme Grant G0600755 (KMM, AJQ), the Research Project of the Ministry of Education, Youth and Sports of the Czech Republic Grant MSM0021620806 (PL, KJ, ME), and the Research Project of the Ministry of Health of the Czech Republic Grant MZOVFN2005 (JZ, MT).

Submitted for publication February 11, 2011; revised June 9, 2011; accepted June 28, 2011.

Disclosure: R.D. Young, None; P. Liskova, None; C. Pinali, None; B.P. Palka, None; M. Palos, None; K. Jirsova, None; E. Hrdlickova, None; M. Tesarova, None; M. Elleder, None; J. Zeman, None; K.M. Meek, None; C. Knupp, None; A.J. Quantock, None

Corresponding author: Andrew J. Quantock, Structural Biophysics Group, School of Optometry and Vision Sciences, Cardiff University, Maindy Road, Cardiff CF24 4LU, Wales, United Kingdom;

quantockaj@cf.ac.uk.
Conclusions. Cellular changes in the MPS VII cornea resemble those in other MPS. However, the wide range of collagen fibril diameters throughout the stroma and the extensive matrix presence of supranormal-sized PG structures appear to be unique features of this disorder. The findings suggest that the accumulation of stromal chondroitin-, dermatan-, and heparansulfate glycosaminoglycans in the absence of $\beta$-glucuronidasemediated degradation can modulate collagen fibrillogenesis. (Invest Ophthalmol Vis Sci. 2011;52:6720-6728) DOI:10.1167/ iovs.11-7377

$T$ he mucopolysaccharidoses (MPS) are a group of lysosomal storage diseases caused by inherited defects in specific enzymes involved in the degradation of glycosaminoglycans (GAGs). ${ }^{1-3}$ The result is a widespread accumulation of dermatan sulfate (DS), chondroitin sulfate (CS), heparan sulfate (HS), or keratan sulfate (KS) in the majority of organs and tissues and a wide range of clinical manifestations. Ocular involvement attributed to GAG deposition in MPS includes progressive corneal clouding, glaucoma, retinal degeneration, and optic disc swelling followed by its atrophy. ${ }^{4}$ In a mouse model of MPS VII, progressive photoreceptor death has also been observed. ${ }^{5}$ The cornea is affected in all the MPS subtypes classified to date, with a significant overlap of corneal phenotype between, and also within, the MPS types and a variety of treatment options. $^{6,7}$

MPS VII (Sly syndrome; Mendelian Inheritance in Man (MIM) \#253220) is a rare, autosomal recessive, inherited condition with an estimated incidence of 1 in 250,000 births, which is one of the lowest of all MPS. Accumulation of undegraded GAGs typically leads to short stature, bone dysplasia, hepatosplenomegaly, hydrops fetalis, and mental retardation. 8,9 The course of the disease is considered clinically attenuated if there is no hydrops fetalis or severe mental retardation and if the condition does not lead to death within a year. ${ }^{8}$ Most children with MPS VII live into teenage or young adult years.

Various approaches toward therapy have been investigated in mutant MPS VII mice, including bone marrow transplantation, ${ }^{10}$ enzyme replacement therapy, ${ }^{11}$ transplacental delivery of the deficient enzyme tagged to Fc into the fetal circulation, ${ }^{12}$ gene therapy, ${ }^{13,14}$ encapsulation, ${ }^{15}$ and stem cell therapy. ${ }^{16}$ However, no effective treatment is yet available for affected human patients. MPS VII is caused by deficiencies of $\beta$-glucuronidase enzyme (GUSB), leading to an accumulation of DS, HS, and CS GAGs in the cells and tissues. ${ }^{17}$ Forty-nine unique disease-causing mutations in the $\beta$-glucuronidase gene (GUSB MIM \#611499) have been identified in persons with MPS VII from various populations. Heterogeneity of pathogenic changes, with different effects on the function of the mutant proteins, manifests as extensive clinical variability among patients with MPS VII. ${ }^{18}$ 
Proteoglycans (PGs) with their GAG side chains, in particular those of the small leucine-rich PG family of molecules, are implicated in the regulation of fibrillar order in cornea. Recent studies on corneal three-dimensional structure using electron tomography have provided new insights into how PGs may help to maintain the ordered architecture of the stroma essential for tissue transparency. ${ }^{19-21}$ Here, we describe a case of MPS VII in which grafting was carried out to treat a reduction of corneal transparency. Clinical, biochemical, molecular genetic, histologic, and ultrastructural examinations of explanted MPS VII corneal tissue were performed, with comparisons with data obtained from a healthy human donor cornea acquired from an eye bank. Data indicate that disturbance in PG turnover resulting from a GUSB deficiency leads to alterations in both fibrillar and interfibrillar components of the corneal stroma.

\section{Materials ANd Methods}

The study conformed to the tenets of the Helsinki Declaration and local ethics codes.

\section{Medical and Ocular History}

The patient reported here was a male, an only child, born to healthy, unrelated parents after an unremarkable pregnancy. Clinical features suggesting MPS diagnosis were present at birth. The $\beta$-glucuronidase activity in isolated leukocytes was $<2 \%$ of controls. Molecular genetic analysis of the GUSB gene revealed compound heterozygosity for two missense mutations, c. $[155 \mathrm{C}>\mathrm{T}]+[1120 \mathrm{C}>\mathrm{T}]$, leading to amino acid changes p.Ser52Phe and p.Arg374Cys. ${ }^{22}$ Medical examination at the age of 24 years revealed short stature $(155 \mathrm{~cm})$, a body weight of $65 \mathrm{~kg}$, and head circumference $61 \mathrm{~cm}$, plus retroflexion of the head with a short neck, sternal prominence, lumbar hyperlordosis, and hepatosplenomegaly. An x-ray survey showed typical signs of dysostosis multiplex. There were thickened aortic and mitral valves on echocardiography and an obstructive type of ventilation with a forced vital capacity of $74 \%$. The urinary GAG excretion, measured on several occasions, was increased between 8 and $25 \mathrm{~g} / \mathrm{mol}$ creatinine compared with a normal value in adults of $1.8 \pm 0.6 \mathrm{~g} / \mathrm{mol},{ }^{23}$ with higher fractions of CS and DS. The patient was not mentally retarded and was able to read, and several IQ tests performed since childhood revealed scores of 81 to 85

At 5 years of age, the patient's best-corrected visual acuity (BCVA) was 0.4 in both eyes and mild corneal clouding was noticed. Refraction was $+4.0 /-5.0 \mathrm{D} \times 7^{\circ}$ in the right eye and $+3.0 /-4.5 \mathrm{D} \times 174^{\circ}$ in the left. At 14 years of age, the patient was referred to the Department of Ophthalmology in Prague with BCVAs of 0.33 (right eye) and 0.4 (left eye). Because of progressive corneal clouding, a penetrating keratoplasty (8-mm diameter button) was performed in the right eye at the age of 20 years and in the left eye at the age of 22 years. Before surgery,
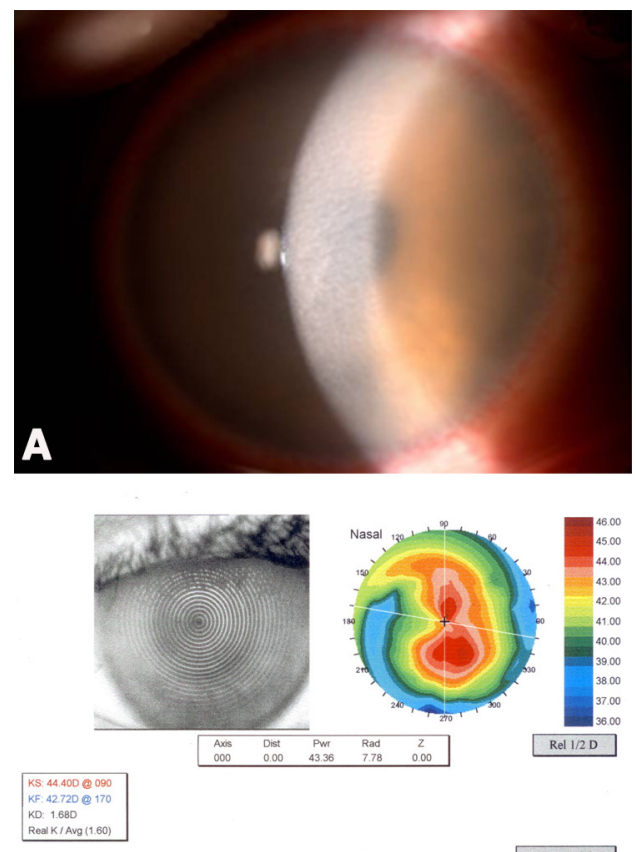

C

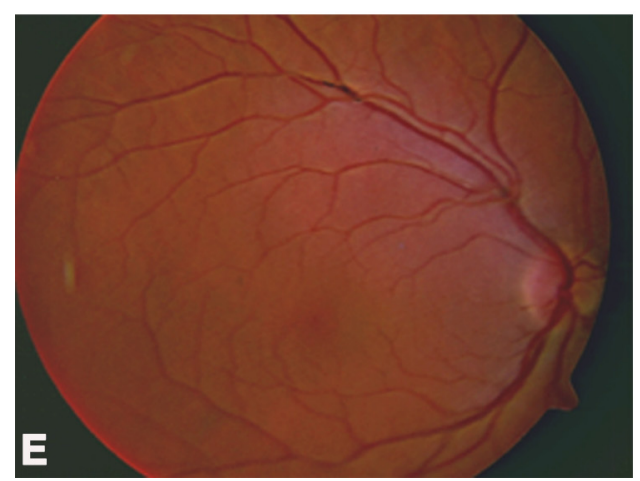

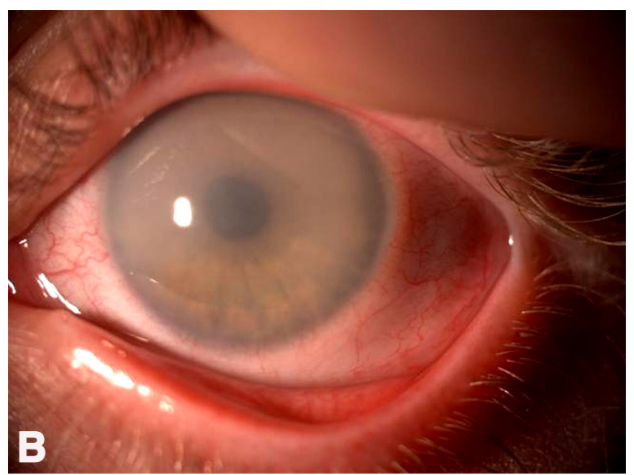
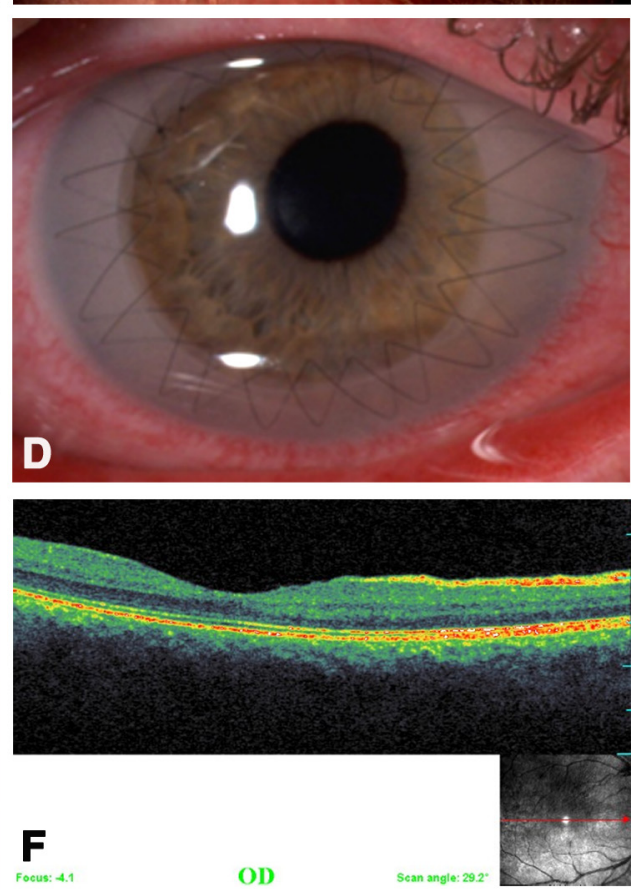

Figure 1. Ocular findings in a patient with mucopolysaccharidosis VII. (A, B) Diffuse fine cloudiness in corneal stroma of the right eye before surgery. (C) Videotopography reveals regular astigmatism in the left eye before grafting. (D) Six months after keratoplasty in the right eye. (E) Normal postoperative appearance of fundus in the right eye. (F) Normal appearance of the macular region on OCT in the right eye.

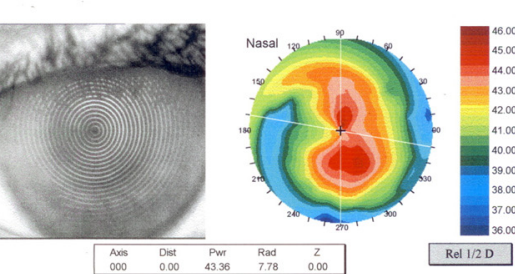
KF. $42.72 D @ 170$
KD. $1.68 D$
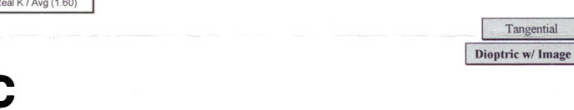

ocus:

Ol) 
diffuse opacification involving all corneal layers was observed bilaterally (Fig. 1), with a BCVA of 0.33 in the right eye and 0.11 in the left. In both eyes the anterior segment was normal, as were intraocular pressure (IOP) measurements, and strabismus was not detected. Regular astigmatism was documented in the left eye (examination not performed in the right eye; Fig. 1). No complications were encountered during or after surgery, and at the last evaluations, 2.5 years and 6 months after right and left keratoplasty, respectively, both corneas remained clear (Fig. 1), with normal IOP and uncorrected visual acuities of 0.25 in the right eye and 0.4 in the left. To the best of our knowledge, there has been only one previous report on corneal transplantation in MPS VII, performed on a 15-year-old patient with mental retardation, which prevented detailed measurement of the visual acuity. ${ }^{24}$ In our patient's right eye, postoperative fundus photography and optical coherence tomography documented, for the first time in an MPS, no abnormalities of the macula area (Fig. 1).

\section{Transmission Electron Microscopy and Electron Tomography}

Immediately after surgery, small (approximately $1 \mathrm{~mm} \times 5 \mathrm{~mm}$ ), full-thickness biopsy samples taken from the center of the left cornea were fixed for 20 hours at room temperature with $2.5 \%$ glutaraldehyde in $25 \mathrm{mM}$ sodium acetate buffer and $0.1 \mathrm{M}$ magnesium chloride, $\mathrm{pH}$ 5.7, which included $0.05 \%$ cuprolinic blue as a cationic stain for sulfated PGs. Tissue was then contrast-enhanced using aqueous, then $50 \%$ ethanolic, $0.5 \%$ sodium tungstate, followed by dehydration in ethanol. For collagen fibril diameter measurements, tissues were prepared by fixation in $2.5 \%$ glutaraldehyde $/ 2 \%$ paraformaldehyde in 0.1 M Sörensen phosphate buffer, $\mathrm{pH} 7.4$, for 3 hours, followed by postfixation in $1 \%$ osmium tetroxide for 1 hour and en bloc contrast in $0.5 \%$ aqueous uranyl acetate and subsequent dehydration. After transfer through propylene oxide, tissue was infiltrated and embedded in Araldite CY212 resin. For fibril diameter measurements, ultrathin sections (90-100 nm thick, cut with a diamond knife) were collected on uncoated G300 copper grids, and stained with saturated aqueous uranyl acetate and lead citrate. For electron tomography of PG ultrastructure, 120-nm-thick sections were collected on G200 grids that were then stained with $1 \%$ aqueous phosphotungstic acid and uranyl acetate. Sections were examined in a transmission electron microscope (JEOL 1010; JEOL, Tokyo, Japan) equipped with a charge-coupled device camera (Orius SC1000; Gatan, Pleasanton, CA).
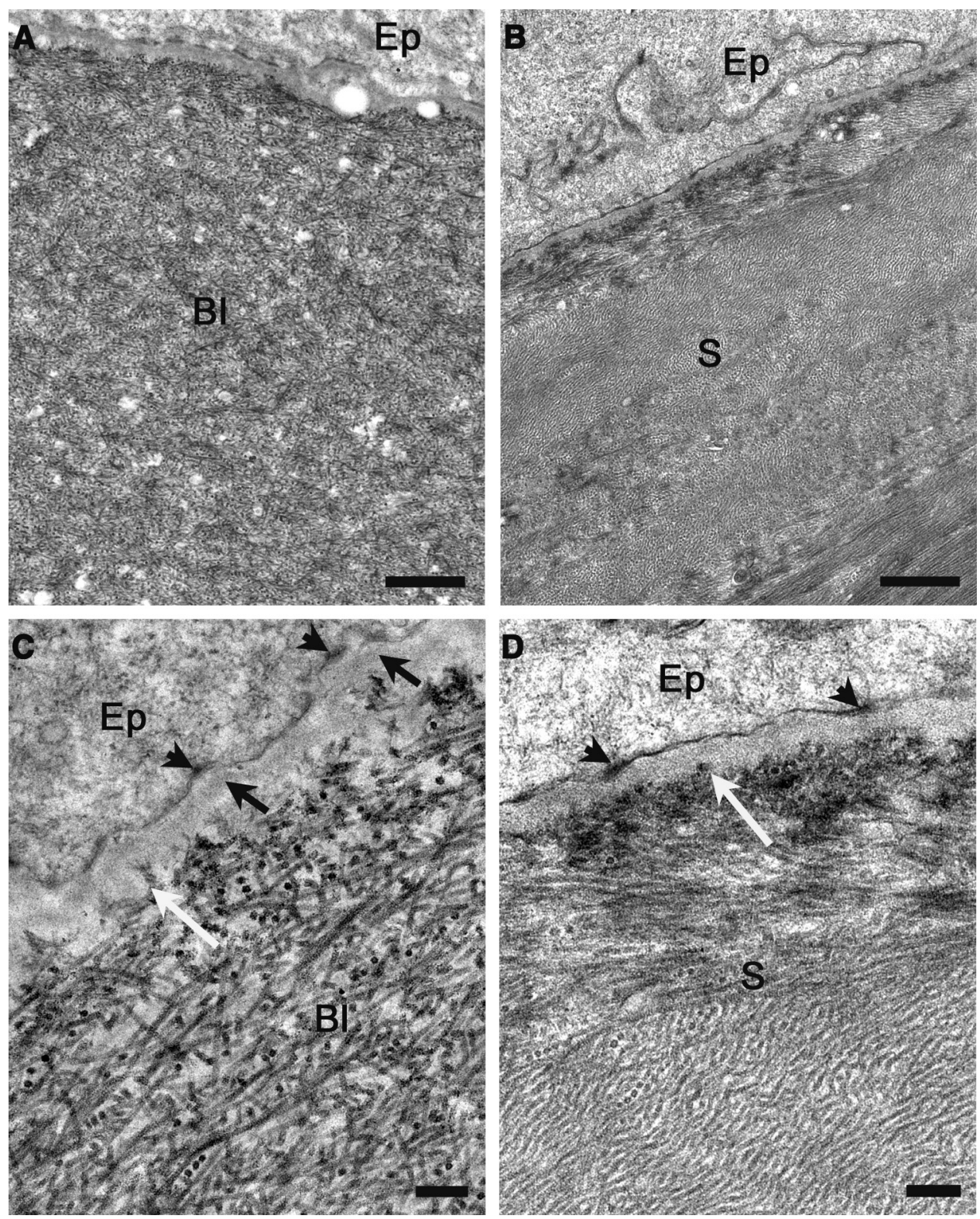

FigURE 2. Electron micrographs of epithelial basement membrane zone in normal $(\mathbf{A}, \mathbf{C})$ and MPS VII cornea $(\mathbf{B}, \mathbf{D})$ cornea. Bowman's layer (Bl) is distinctive in normal cornea $(\mathbf{A}, \mathbf{C})$ but poorly defined, or absent, in MPS VII (B, D). Anchoring fibrils (white arrow) appear to insert directly into superficial stroma (S) in MPS VII (D) rather than into Bowman's layer as in normal cornea (C). Basal epithelial cells (Ep) exhibit hemidesmosomes (C, D; arrowheads). Basal lamina, distinctive in normal cornea (black arrows), appears diffuse in MPS VII with an underlying granular deposit (D). Scale bars, $1 \mu \mathrm{m}$ (A, B); $200 \mathrm{~nm}(\mathbf{C}, \mathbf{D})$. 
For comparison with data obtained from the MPS VII cornea, specimens of normal human cornea were also obtained and prepared by the same methods of fixation. A left-right pair of human corneas, unsuitable for use in transplantation, was acquired for research purposes from the Northwest Lions Eye Bank (Seattle, WA) with full informed consent and institutional approval. These tissues were the subject of an earlier published study ${ }^{25}$ that details, in full, methods of preparation before fixation.

Fibril diameter measurements were made on electron micrographs, taken at $20,000 \times$ magnification, of the corneal tissue that had been postfixed with osmium tetroxide. ImageJ software (developed by Wayne Rasband, National Institutes of Health, Bethesda, MD; available at http://rsb.info.nih.gov/ij/index.html) was used, with the measuring tool calibrated against micrograph scale bars generated by imaging software (Digital Micrograph; Gatan) and derived from images of a 2160 lines/mm diffraction grating acquired with the charge-coupled device camera (Orius SC1000; Gatan). Areas were selected in which fibrils appeared in sharply defined transverse sections. Straight-line values were taken across the shortest diameter for each individual fibril to minimize any error where the section plane might have deviated from true transverse section and where irregular fibril profiles were encountered. Histograms of fibril diameters were prepared using predictive analysis (SPSS 16; IBM, Armonk, NY).

For electron tomography, $10 \mathrm{~nm}$ colloidal gold particles were added to both sides of the stained 120-nm-thick sections of cuprolinic blue-contrasted tissue. The gold particles served as fiducial markers to facilitate the alignment of a series of 60 images taken at $20,000 \times$ magnification and at $2^{\circ}$ tilt increments from $-60^{\circ}$ to $+60^{\circ}$. The image sequence was processed through IMOD, ImageJ, and EM3D software to generate a three-dimensional reconstruction of collagen-PG associations viewed in longitudinal section and was achieved according to previously described techniques. ${ }^{19-21}$

\section{Results}

The MPS VII cornea was surfaced by a normal, stratified epithelium with hemidesmosomes visible on proximal membranes of the basal cells (Fig. 2). However, the epithelial basal lamina appeared diffuse compared with that in normal cornea (Figs. 2C, 2D). An underlying granular deposit enveloped anchoring fibrils that apparently inserted directly into superficial stroma, as Bowman's layer appeared undeveloped or absent (Figs. 2B, 2D). Stromal keratocytes, typical of fibroblasts in lysosomal storage disease, were mostly enlarged and characterized by conspicuous distension of the lysosomal system by whorl-like or granular contents of variable electron density (Figs. 3A-D). All cells throughout the full thickness of the corneal stroma appeared affected, though occasionally individual cells were located with minimal, or preliminary, indications of lysosomal accumulation (Fig. 3B). Keratocyte cytoplasmic vacuole contents bound the cationic dye cuprolinic blue, indicating intracellular sequestration of polyanionic material presumed to be PGs (Fig. 3E). In some cells, empty lucent vacuoles suggested extracellular release of contents by degenerating keratocytes (Figs. 3A, 3D). Intrastromal vacuoles contained complexed dye (Fig. 3F), also implying the release of compartmentalized PG or GAG cargo from cells into the matrix, although whether these structures were membrane bound could not be conclusively demonstrated. Descemet's membrane and endothelial cells exhibited no signs of lysosomal storage.

Stromal lamellae appeared compact in the MPS VII cornea, but, unlike stromal lamellae in normal cornea (Figs. 4A, 4C, $4 \mathrm{E})$, wide variations in collagen fibril diameter were evident when viewed in transverse section and were readily located at
FIGURE 3. Keratocyte morphology and granular accumulation in MPS VII cornea. (A) Most keratocytes throughout the corneal thickness, as shown here in mid stroma, exhibit evidence of lysosomal storage with an accumulation of cytoplasmic vacuoles. Scale bar, $2.5 \mu \mathrm{m}$. (B) Occasional cells with minimal vacuole accumulation are present. Scale bar, $1 \mu \mathrm{m}$. (C, D) Cytoplasmic vacuoles appear with dense granular contents or empty after extraction or release of contents. Scale bar, $1 \mu \mathrm{m}$. (E) Intracellular cuprolinic blue labeling (arrowheads) indicates polyanionic material retained within cellular vacuoles. Scale bar, 1 $\mu \mathrm{m}$. (F) Vacuoles and cuprolinic bluelabeled contents may also be released into the extracellular matrix. Scale bar, $250 \mathrm{~nm}$.
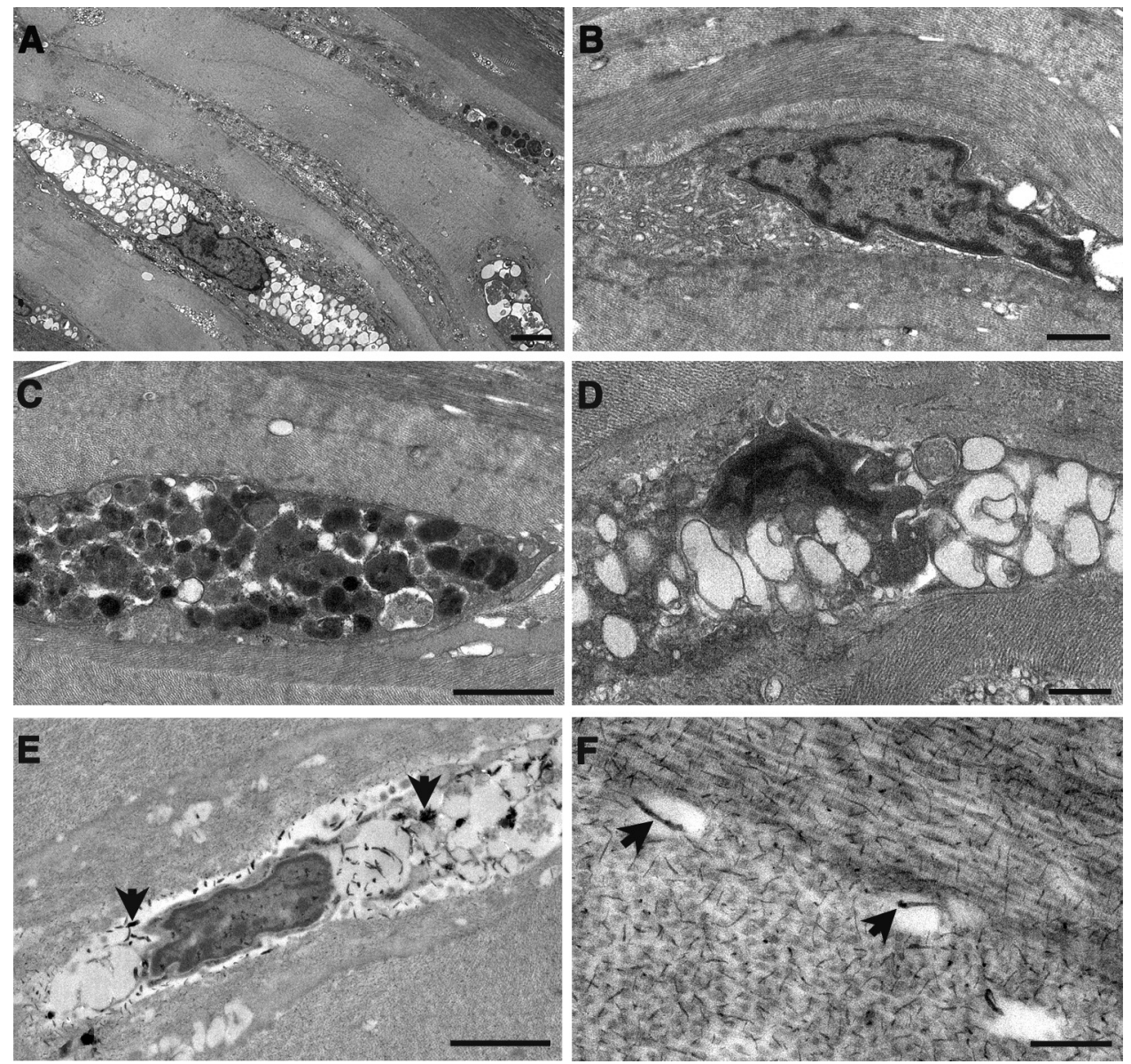

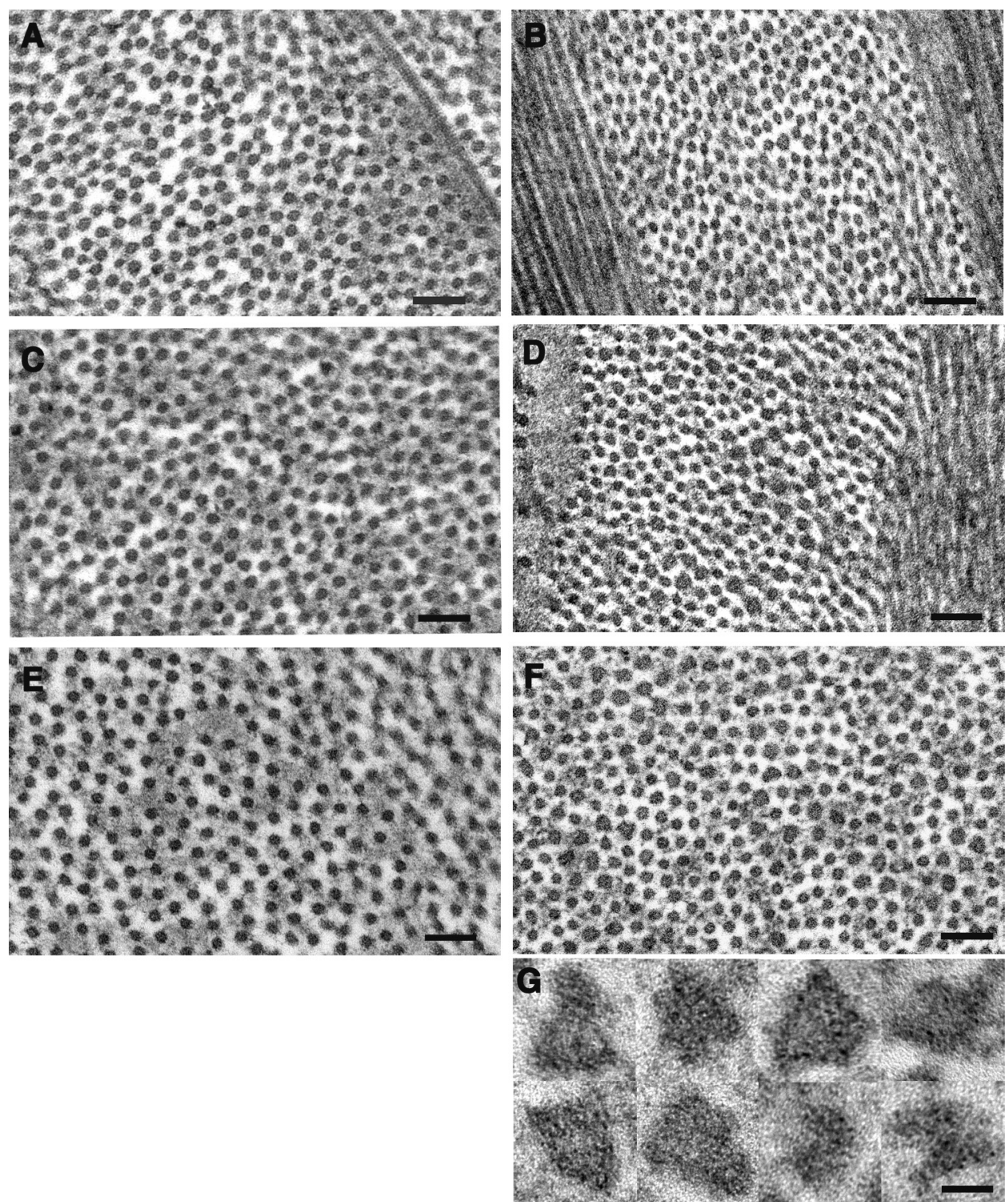

FIGURE 4. Stromal ultrastructure. Collagen fibrils in transverse section in normal (A, C, E) and MPS VII (B, D, F) cornea at locations in anterior $(\mathbf{A}, \mathbf{B})$, mid $(\mathbf{C}, \mathbf{D})$, and posterior $(\mathbf{E}, \mathbf{F})$ stroma. Fibrils show wide variation in diameter in MPS VII throughout the stromal thickness, appearing generally smaller overall than in normal tissue. Scale bars, $100 \mathrm{~nm}$. (G) In the MPS VII cornea, deviation from the circular profile of some fibrils in transverse section suggests less regulated control of fibril shape than in the normal cornea. Scale bar, $25 \mathrm{~nm}$. all depths throughout the stroma (Figs. 4B, 4D, 4F). In addition, fibrils with irregular profiles were prevalent in the MPS VII stroma (Fig. 4G). In contrast to normal cornea, accumulations of long-spaced collagen appeared abundant in MPS VII.

Measurements of collagen fibrils in the control eye bank cornea examined here showed a statistically normal distribution of fibril diameters throughout the stroma (Figs. 5A, 5C, $5 \mathrm{E}$ ), with a trans-stromal mean of $23.53 \mathrm{~nm}$ compared with published mean values of $26.0 \mathrm{~nm} .{ }^{26}$ Fibril diameters ranged from 14.6 to $29.0 \mathrm{~nm}$ (mean $23.0 \mathrm{~nm}, \mathrm{SD} \pm 2.1 \mathrm{~nm} ; n=1278$ ), in the anterior stroma, to 15.8 to $31.0 \mathrm{~nm}$ (mean $23.9 \mathrm{~nm}, \mathrm{SD} \pm$ $2.0 \mathrm{~nm} ; n=1268$ ), in the mid stroma, and to 17.3 to $32.0 \mathrm{~nm}$ (mean $23.8 \mathrm{~nm}, \mathrm{SD} \pm 2.0 \mathrm{~nm} ; n=1093$ ) in the posterior stroma. In the MPS VII cornea, collagen fibrils appeared regularly packed, although fibrils were not of uniform diameter (Figs. 4, 5). Small and large collagen fibrils were visible throughout the stromal thickness, with abnormal diameters especially prominent in the mid and posterior stroma (Fig. 5). Measurements of fibril diameter at different depths in MPS VII showed a broader range than was seen in normal cornea. Fibril diameters ranged from $13.6 \mathrm{~nm}$ to $32.2 \mathrm{~nm}$ in the anterior stroma ( $n=1104)$, from $12.7 \mathrm{~nm}$ to $41.6 \mathrm{~nm}$ in the mid stroma $(n=1026)$, and from $16.6 \mathrm{~nm}$ to $38.8 \mathrm{~nm}$ in the posterior stroma ( $n=1136$; Figs. 5B, 5D, 5F). A one-sample Kolmogorov-Smirnov test showed that in the MPS VII cornea, none of the fibril diameter distributions was normal at any of the stromal depths examined (anterior, $P \leq 0.001$; mid, $P \leq 0.000$; posterior, $P \leq 0.000)$. In the anterior stroma, the spectrum of collagen fibril diameters was not as broad as in the mid and posterior stroma but still differed significantly from the normal cornea. Fibrils in the mid stroma showed indications of a bimodal distribution of diameter measurements (Fig. 5D).

Proteoglycans visualized by cuprolinic blue staining in this case of MPS VII appeared different from those in the normal cornea (Fig. 6). In contrast to the fine filamentous or rounded structures radiating from collagen fibrils in normal cornea (Figs. 6A, 6B, 6E, 6F, 6I, 6J), they assumed a more elongated, thicker, and more electron-dense appearance (Figs. 6C, 6D, $6 \mathrm{G}, 6 \mathrm{H}, 6 \mathrm{~K}, 6 \mathrm{~L}$ ), apparently traversing several collagen fibrils (Figs. 6G, 6K). These PG-dye complexes rarely exhibited regular D-periodic association with the fibrils or any interactions with long-spacing collagen. Three-dimensional reconstruction from electron tomography of collagen fibrils in longitudinal section in MPS VII clearly showed that PGs traversed up to four collagen fibrils and were also visible running axially along 
FIGURE 5. Histograms showing measurements of collagen fibril diameters in normal $(\mathbf{A}, \mathbf{C}, \mathbf{E})$ and MPS VII $(\mathbf{B}, \mathbf{D}$, F) cornea. Anterior stromal collagen fibril diameters are given here. (A) Normal cornea: fibril diameter range, 14.6 to $29.0 \mathrm{~nm}$; mean, $23.0 \mathrm{~nm}, \mathrm{SD} \pm$ $2.1 \mathrm{~nm}(n=1278)$. (B) MPS VII cornea: fibril diameter range, 13.6 to 32.2 $\mathrm{nm}(n=1104)$. Mid stromal collagen fibril diameters are given here. (C). Normal cornea: fibril diameter range, 15.8 to $31.0 \mathrm{~nm}$; mean, $23.9 \mathrm{~nm}, \mathrm{SD} \pm$ $2.0 \mathrm{~nm}(n=1268)$. (D) MPS VII cornea: fibril diameter range, 12.7 to 41.6 $\mathrm{nm}(n=1026)$. Posterior stromal collagen fibril diameters are given here. (E) Normal cornea: fibril diameter range, 17.3 to $31.9 \mathrm{~nm}$; mean, 23.8 $\mathrm{nm}, \mathrm{SD} \pm 2.04 \mathrm{~nm}(n=1093)$. (F) MPS VII cornea: fibril diameter range, 16.6 to $38.8 \mathrm{~nm}(n=1136)$.
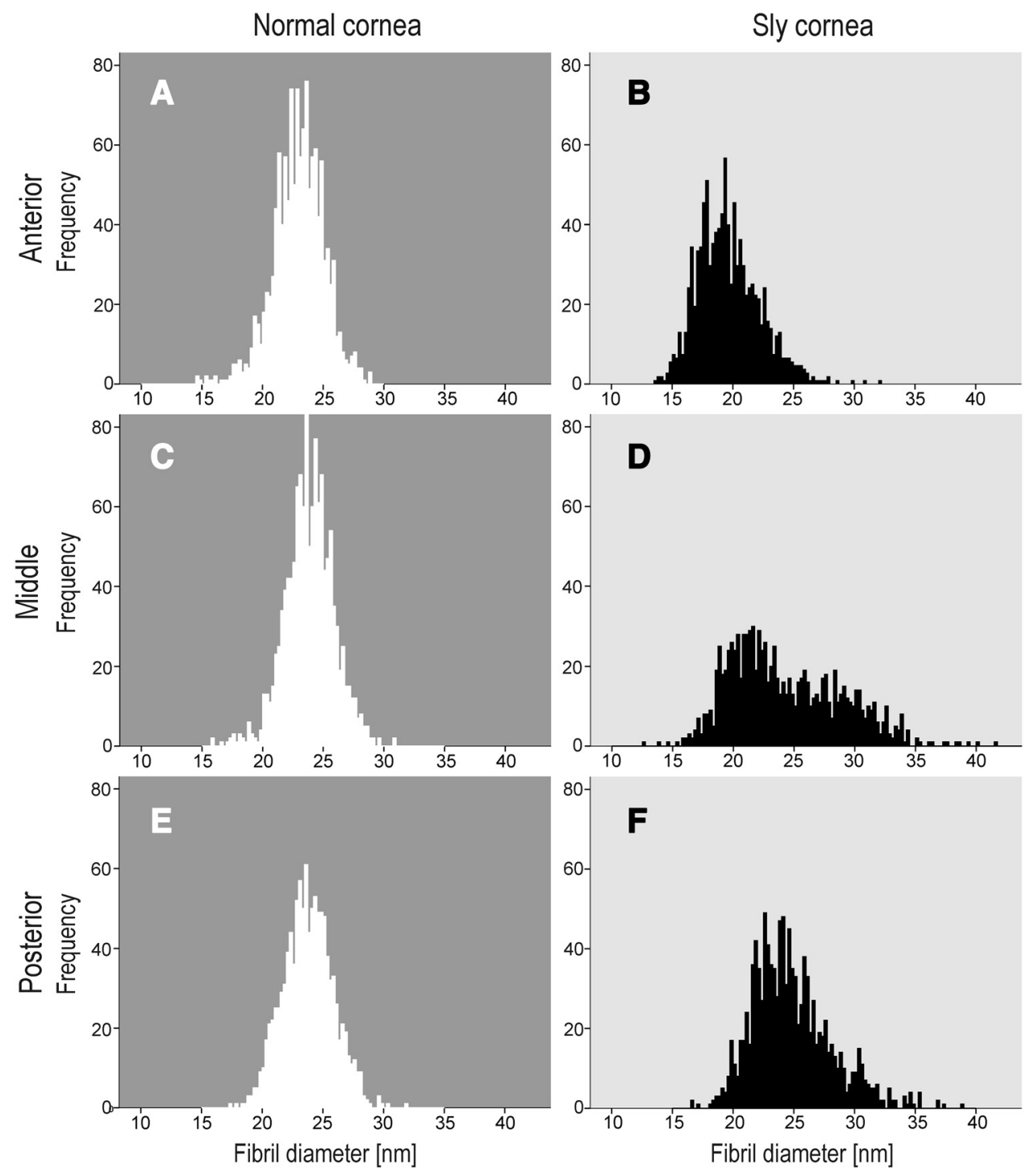

fibrils (Fig. 7 and Supplementary Movie S1, http://www.iovs. org/lookup/suppl/doi:10.1167/iovs.11-7377/-/DCSupplemental).

\section{Discussion}

Since its identification more than 30 years ago, ${ }^{27}$ the extreme rarity of MPS VII has meant that investigations into its genetic origins and phenotypic manifestations have been few. Variations in the severity of clinical symptoms between individual cases in MPS VII, in contrast, have been well documented, ${ }^{3}$ and in milder forms of the disease $10 \%$ of normal levels of $\beta$-glucuronidase has been demonstrated in cultured fibroblasts. ${ }^{28}$ Hence, some turnover, albeit reduced, of its substrates DS, CS, and HS GAGs is still present. It should be emphasized, therefore, that the pathologic features we describe in this single case of MPS VII cornea may not necessarily be representative of all disease variants, though comparisons with normal cornea illustrate significant differences in matrix ultrastructure. The pathogenic variants in our case are a very rare cause of MPS VII. The c. $155 \mathrm{C}>\mathrm{T}$ change located in exon 1 has been found only in our patient, whereas c. $1120 \mathrm{C}>\mathrm{T}$ has been further detected in one allele of a German proband. ${ }^{8}$ It is predicted that the phenotypic severity in our patient is predominantly influenced by the c. $155 \mathrm{C}>\mathrm{T}$ mutation, which causes total destruction of $\beta$-glu- curonidase enzyme activity in transfected cells. The c. $1120 \mathrm{C}>\mathrm{T}$ mutation leads to a residual activity of $5.5 \% \mathrm{com}-$ pared with the wild type, ${ }^{28}$ which, in this patient, might have led to an absence of the mental retardation usually observed in patients with MPS VII.

Recently, our understanding of $\beta$-glucuronidase deficiency in MPS VII has been advanced by studies of model systems in animals. ${ }^{29}$ However, tissue studies of ocular manifestations of the clinical disease in humans, particularly in the cornea, have been limited by the infrequent availability of surgical specimens from keratoplasty. ${ }^{24}$ Consequently, to our knowledge, there have been no previous reports of corneal matrix changes at the fibrillar level arising from $\beta$-glucuronidase-deficient GAG turnover in MPS VII. Bergwerk et al. ${ }^{24}$ showed vacuoles and inclusions in keratocytes by electron microscopy in MPS VII but made no mention of alterations in collagen and PG organization of the extracellular matrix such as those we describe, and that, along with the presence of abnormal cells in the stroma, were likely related to the loss of transparency. Progressive corneal opacification affects all forms of MPS, ${ }^{6,7}$ and although the severity of the opacification differs across these conditions, there is a common appearance of corneal keratocytes, as we describe here in MPS VII, with a massed accumulation of GAGs in the endosomal-lysosomal compart- 

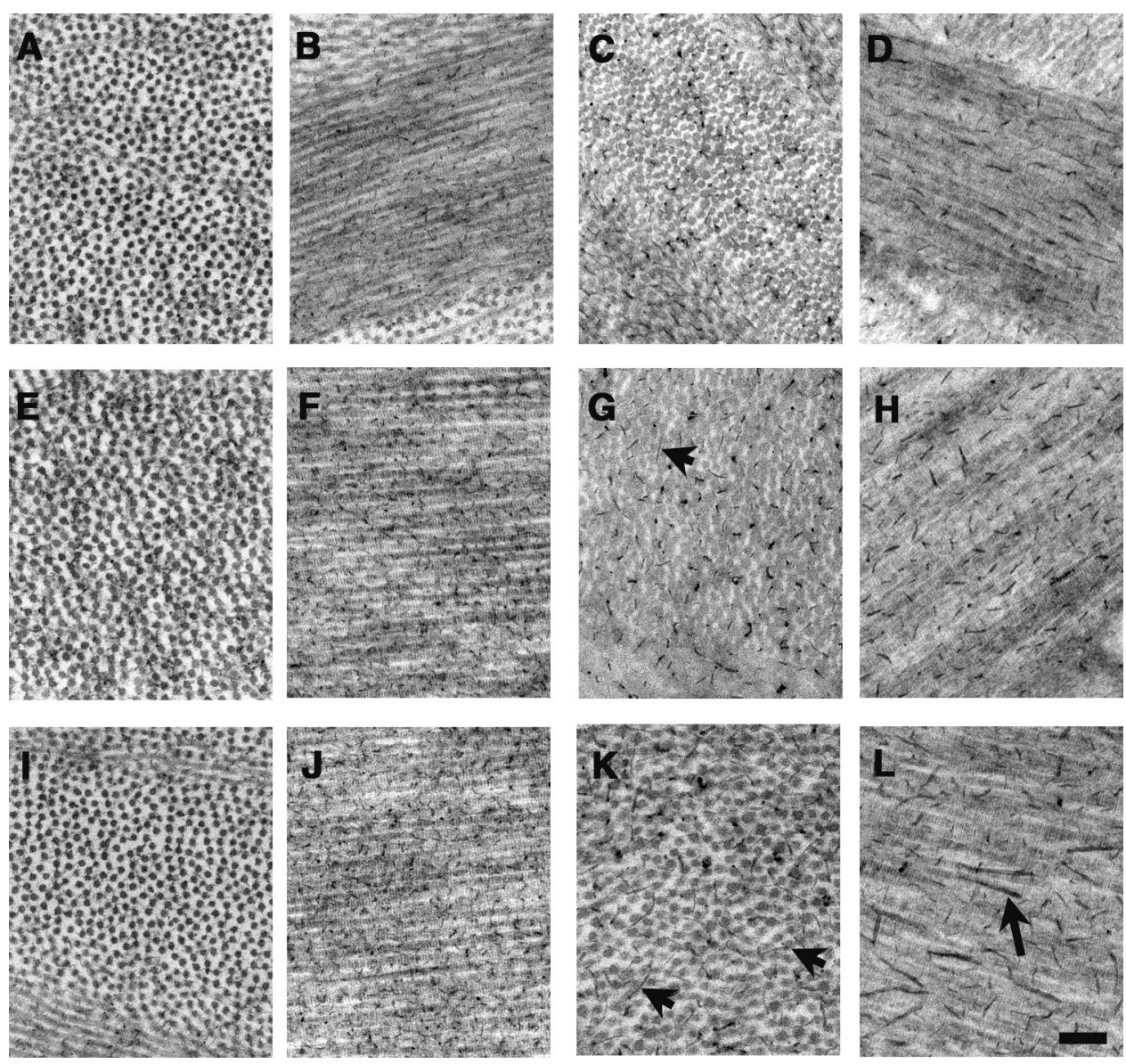

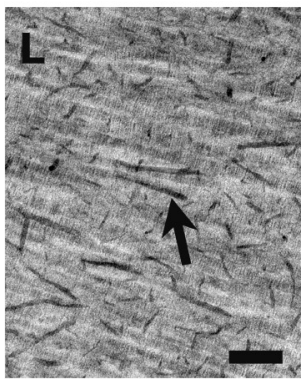

FiguRE 6. Proteoglycans visualized by cuprolinic blue in normal cornea (two left-band columns) and MPS VII cornea (two right-hand columns) in anterior (top row), mid (central row), and posterior (bottom row) stroma. In normal cornea, proteoglycans appear as fine filaments radiating from collagen fibrils in transverse (A, E, I) and longitudinal $(\mathbf{B}, \mathbf{F}, \mathbf{J})$ section. In MPS cornea, filaments appear larger in transverse $(\mathbf{C}, \mathbf{G}, \mathbf{K})$ and longitudinal $(\mathrm{D}, \mathbf{H}, \mathbf{L})$ section. Filaments traverse several collagen fibrils (G, K; arrowheads) and sometimes run axially over several D-periods (L; arrow). Scale bar, $150 \mathrm{~nm}$. ment of the cells. Similar cellular pathology occurs, for example, in Morquio syndrome (MPS IV), in which the degradation of KS GAG is impaired and in which, as documented here in MPS VII, alterations in epithelial basement membrane and aberrant Bowman's layer are also present. ${ }^{30,31}$ Cuprolinic bluestained deposits have also been reported inside keratocytes in MPS I-H (Hurler syndrome), ${ }^{32}$ in which $\alpha$-L-iduronidase deficiency leads to an accumulation of HS and DS, and in MPS VI
(Maroteaux-Lamy syndrome), ${ }^{33}$ in which DS accumulates as a result of $\mathrm{N}$-acetylgalactosamine 4-sulfatase deficiency.

In addition to the changes in keratocyte morphology indicative of overload on the lysosomal degradation pathways for GAGs, the corneal stroma in MPS contains supranormal levels of the partially degraded molecules, variously described as granular or membranous material. ${ }^{24,30,31}$ It is likely that excess extracellular GAG may arise both from newly secreted material

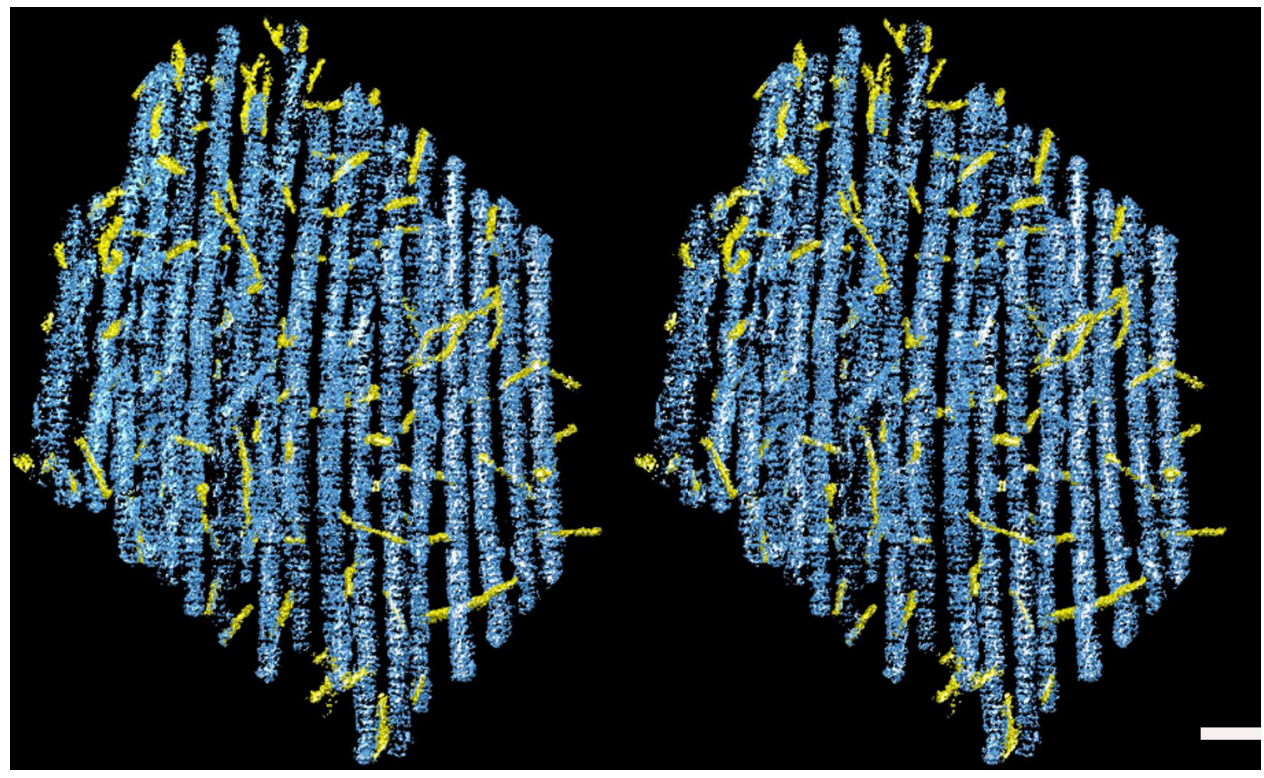

FigURE 7. 3D reconstruction of collagen fibrils, shown here in longitudinal section, and associated proteoglycans in posterior stroma of MPS VII cornea. Collagen, false-colored blue, proteoglycans, yellow. Proteoglycans both traverse and run axially with respect to fibril orientation and link up to four collagen fibrils. Scale bar, $100 \mathrm{~nm}$. To obtain a 3D impression from the stereo paired images, display images approximately 6 to $7 \mathrm{~cm}$ apart and apply your nose onto the page/screen between the two images. Keeping both eyes open, view the two out-of-focus images superimposed; slowly move to 30 to $40 \mathrm{~cm}$ from eye to the page/screen; when you can focus, you should see three images. The middle one should have appreciable depth; ignore the flat images on either side. 
and from incomplete degradation products of the extracellular matrix. Although more than 50\% of newly synthesized PGs are thought to be degraded through the lysosomal system in normal tissues, without extracellular secretion, in MPS, partially degraded intracellular GAG breakdown fragments could potentially be trafficked into the extracellular space. One clear route for this appears to be through the degeneration of keratocytes and the release of storage vacuoles pericellularly, after disruption of the plasma membrane. Alternatively, increased intracellular calcium associated with membrane damage has been shown to lead to lysosomal exocytosis, with up to $24 \%$ involvement of peripheral lysosomes in primary fibroblasts in vitro. ${ }^{34}$ Degradation pathways of PGs in the matrix outside the cell are also incompletely understood. Small PGs are probably released from their collagen associations by the activity of extracellular metalloproteases on their protein cores, ${ }^{35}$ after which they are taken into the cells by endocytosis for sequential breakdown in the lysosomal system by endoglycosidases, exoglycosidases, and sulfatases. ${ }^{36}$ The receptor-mediated process by which small PGs, including decorin and lumican, either as whole PGs or as partially digested fragments enter the cell may include binding to Toll-like receptors now known to be present on corneal cells ${ }^{37}$ and on phagocytes of the immune system. This route is accepted as a potential conduit toward mediation of inflammatory and immune responses in keratocytes, ${ }^{38}$ through transformation to myofibroblasts, and by mononuclear cells.

The participation of $\beta$-glucuronidase in GAG degradation occurs at a relatively late stage in lysosomal degradation of GAGs to produce small glycan fragments still carrying sulfate groups. Accumulation of these, together with larger moieties released from the matrix by proteolytic degradation, presumably gives rise to a heterogeneous population of sulfated CS, DS, and HS GAGs present as free matrix components in MPS VII, which would be available for association by electrostatic interaction with other structural components in the stroma. We report here cuprolinic blue-complexed PG structures in MPS VII cornea that greatly exceed in length those previously reported in normal human corneas or indeed those in other species. PGs in MPS VII often traversed multiple collagen fibrils, sometimes up to four in number, and were either orientated axially along fibrils or weaved through the extrafibrillar space laterally to the fibrils. Large cuprolinic bluepositive structures have also been documented in the corneas of Chst 5 null mice, where they are branched, and are assumed to be aggregated CS/DS proteoglycans. ${ }^{39,40}$ In a previous study ${ }^{41}$ on Morquio syndrome in which turnover of KS GAG is impaired, PG filaments visualized with cuprolinic blue also appeared increased in length. Abnormally large cuprolinic blue-positive structures were found as well in the stroma of $\alpha$-L-iduronidase-deficient MPS I-H (Hurler syndrome) ${ }^{32}$ and MPS I-S (Scheie syndrome) corneas. ${ }^{26}$ These observations support the concept that excessive free GAGs in the matrix have the capacity to self-aggregate or to bind to existing matrix constituents to generate supranormal-sized structures. Overall, however, dye-complexed PGs in MPS VII, though larger, appeared fewer in number than in normal cornea and without abundant D-periodic collagen associations. Although evidence is not available here, small leucine-rich proteoglycans may be able to substitute functionally when the absence, or overproduction, of one molecule might lead to an imbalance. ${ }^{42,43}$ Thus compensatory downregulation of KS and KS-bearing core proteins may occur in MPS VII, where matrix accumulation of CS and DS leads to the formation instead of larger PG aggregates. This may explain the apparent scarcity of small fibril-associated cuprolinic blue-stained filaments, which in the cornea are presumed to be KS PGs in the MPS VII stroma.

The discovery of smaller than normal collagen fibril diameters in MPS VII reported here is consistent with indications that increased amounts of decorin result in smaller fibrils. ${ }^{44} \mathrm{How}$ ever, though the enzyme defect in MPS VII would be expected to cause an increase in DS as well as in CS and HS GAGs in the matrix, whether core protein concentrations would also be increased is unknown. Reduced fibril diameter was detected throughout the stromal thickness, which is not surprising because decorin and DS/CS concentrations are not known to vary with stromal depth. Alterations in collagen fibril morphology have been reported previously in MPS I, IV, and VI, all of which exhibited corneal opacities, but in all these the average fibril diameter was increased. ${ }^{26,32,41,45}$ Bimodal distribution of fibril diameter measurements, as detected in the mid stroma here, was also previously reported in MPS IS (Scheie), ${ }^{26}$ MPS IV (Morquio), ${ }^{41}$ and MPS IV (Hurler) ${ }^{32}$ and was interpreted as fibril aggregation. The presence of irregular fibril profiles in the MPS VII cornea suggests less regulated control of fibril shape than in normal cornea. However, this is difficult to reconcile with current evidence that indicates the deficiency of decorin $^{46,47}$ and of other small leucine-rich proteoglycans ${ }^{48}$ has been linked to aberrations in fibril form through uncontrolled lateral fibril growth in the absence of binding of these molecules to the fibril surface. The mechanisms whereby excess matrix DS, CS, and HS GAGs in MPS VII might lead to dysregulation of fibril shape are unknown.

MPS syndromes, including MPS VII, through deficiencies in enzymes involved in GAG turnover, lead to a range of alterations in both interfibrillar and fibrillar extracellular matrix components of the cornea and yet have a common outcome in loss of corneal function through stromal opacity. They emphasize the complexities of interactions between matrix components and the deficiencies in our knowledge of their functions and pathways of remodeling. Further studies of these rare conditions and of animal models that mimic their enzyme defects should greatly facilitate an understanding of the functional role of corneal components and their intermolecular organization. In addition, clarification of the relationship between genotype and the clinical and microscopic manifestations of MPS VII should provide useful information for the development of effective molecular therapies.

\section{Acknowledgments}

The authors thank Emyr Lloyd-Evans (School of Biological Sciences, Cardiff University) for helpful discussion on membrane trafficking in the endosomal-lysosomal system.

\section{References}

1. Neufeld EF, Muenzer J. The mucopolysaccharidoses. In: Scriver CR, Beaudet AL, Sly WS, Valle D, eds. The Metabolic and Molecular Bases of Inberited Disease. Vol. 8. New York: McGraw-Hill; 2001:3421-3452.

2. Biswas J, Nandi K, Sridharan S, Ranjan P. Ocular manifestations of storage diseases. Curr Opin Opbthalmol. 2008;19:507-511.

3. Ashworth JL, Kruse FE, Bachmann B, et al. Ocular manifestations in the mucopolysaccharidoses: a review. Clin Exp Opbthalmol. 2010; 38:12-22.

4. Collins ML, Traboulsi EI, Maumenee IH. Optic nerve head swelling and optic atrophy in the systemic mucopolysaccharidoses. $O p h$ thalmology. 1990;97:1445-1449.

5. Lazarus HS, Sly WS, Kyle JW, et al. Photoreceptor degeneration and altered distribution of interphotoreceptor matrix proteoglycans in the mucopolysaccharidosis VII mouse. Exp Eye Res. 1993;56:531541.

6. Ashworth JL, Biswas S, Wraith E, Lloyd IC. Mucopolysaccharidoses and the eye. Surv Ophthalmol. 2006;51:1-17.

7. Klintworth GK, Jester JV. Genetic basis of corneal diseases and the role of keratocytes in corneal transparency: a review. Clin Exp Ophthalmol. 2010;38:23-33. 
8. Vervoort R, Islam MR, Sly WS, et al. Molecular analysis of patients with $\beta$-glucuronidase deficiency presenting as hydrops fetalis or as early mucopolysaccharidosis VII. Am J Hum Genet. 1996;58:457471.

9. Cheng Y, Verp MS, Knutel T, Hibbard JU. Mucopolysaccharidosis type VII as a cause of recurrent nonimmune hydrops fetalis. $J$ Perinat Med. 2003;31:535-537.

10. Birkenmeier EH, Barker JE, Vogler CA, et al. Increased life span and correction of metabolic defects in murine mucopolysaccharidosis type VII after syngeneic bone marrow transplantation. Blood. 1991;78:3081-3092.

11. Vogler C, Levy B, Galvin NJ, et al. Enzyme replacement in murine mucopolysaccharidosis type VII: neuronal and glial response to beta-glucuronidase requires early initiation of enzyme replacement therapy. Pediatr Res. 1999;45:838-844.

12. Grubb JH, Vogler C, Tan Y, Shah GN, MacRae AF, Sly WS. Infused Fc-tagged $\beta$-glucuronidase crosses the placenta and produces clearance of storage in utero in mucopolysaccharidosis VII mice. Proc Natl Acad Sci US A. 2008;105:8375-8380.

13. Wolfe JH, Sands MS, Barker JE, et al. Reversal of pathology in murine mucopolysaccharidosis type VII by somatic cell gene transfer. Nature. 1992;360:749-753

14. Ponder KP, Auricchio A. Gene therapy for ocular problems in mucopolysaccharidosis: an experimental and promising approach with benefits in animal models-a review. Clin Exp Ophthalmol. 2010;38:43-51

15. Nakama H, Ohsugi K, Otsuki T, et al. Encapsulation cell therapy for mucopolysaccharidosis type VII using genetically engineered immortalized human amniotic epithelial cells. Toboku J Exp Med. 2006;209:23-32.

16. Pinello L, Busin M, Fontana L, Dua HS. Application of (lamellar) keratoplasty and limbal stem cell transplantation for corneal clouding in the mucopolysaccharidoses-a review. Clin Exp Opbthalmol. 2010;38:52-62.

17. Tomatsu S, Montano AM, Dung VC, Grubb JH, Sly WS. Mutations and polymorphisms in GUSB gene in mucopolysaccharidosis VII (Sly syndrome). Hum Mutat. 2009;30:511-519.

18. Lorincz AE. The mucopolysaccharidoses: advances in understanding and treatment. Pediatr Ann. 1978;7:104-122.

19. Knupp C, Pinali C, Lewis PN, et al. The architecture of the cornea and structural basis for its transparency. Adv Prot Chem Struct Biol. 2009;78:25-49.

20. Lewis PN, Pinali C, Young RD, Meek KM, Quantock AJ, Knupp C. Structural interactions between collagen and proteoglycans are elucidated by three-dimensional electron tomography of bovine cornea. Structure. 2010;18:239-245

21. Parfitt GJ, Pinali C, Young RD, Quantock AJ, Knupp C. Threedimensional reconstruction of collagen-proteoglycan interactions in the mouse corneal stroma by electron tomography. J Struct Biol. 2010;170:392-397.

22. Vervoort R, Buist NR, Kleijer WJ, et al. Molecular analysis of the beta-glucuronidase gene: novel mutations in mucopolysaccharidosis type VII and heterogeneity of the polyadenylation region. Hum Genet. 1997;99:462-468.

23. Thuy LP, Nyhan WL. A new quantitative assay for glycosaminoglycans. Clin Chim Acta. 1992;212:17-26.

24. Bergwerk KE, Falk RE, Glasgow BJ, et al. Corneal transplantation in a patient with mucopolysaccharidosis type VII (Sly disease). Ophthalmic Genet. 2000;21:17-20.

25. Quantock AJ, Boote C, Young RD, et al. Small-angle fibre diffraction studies of corneal matrix structure $J$ Appl Cryst 2007;40: S335-S340.

26. Quantock AJ, Meek KM, Fullwood NJ, Zabel RW. Scheie's syndrome: the architecture of corneal collagen and distribution of corneal proteoglycans. Can J Opbthalmol. 1993;28:266-272.

27. Sly WS, Quinton BA, McAlister WH, Rimoin DL. Beta glucuronidase deficiency: report of clinical, radiologic and biochemical features of a new mucopolysacchaidosis. J Pediatrics. 1973;82:249-257.

28. Gitzelmann R, Wiesmann UN, Spycher MA, Herschkowitz N, Giedion A. Unusually mild course of B-glucuronidase deficiency in two brothers (mucopolysaccharidosis VII). Helv Pediatr Acta. 1978:33:413-428.
29. Mollard RJ, Telegan P, Haskins M, Aguirre G. Corneal endothelium in mucopolysaccharide storage disorders: morphologic studies in animal models. Cornea. 1996;15:25-34.

30. Iwamoto M, Nawa Y, Maumenee IH, Young-Ramasaran J, Matalon $\mathrm{R}$, Green WR. Ocular histopathology and ultrastructure of Morquio syndrome (systemic mucopolysaccharidosis IVA). Graefes Arch Clin Exp Opbthalmol. 1990;228:342-349.

31. Leslie T, Siddiqui MAR, Aitken DA, Kirkness CM, Lee WR. Morquio syndrome: electron microscopic findings. Br J Ophthalmol. 2005; 89:925-926.

32. Huang Y, Bron AJ, Meek KM, Vellodi A, McDonald B. Ultrastructural study of the cornea in a bone marrow-transplanted Hurler syndrome patient. Exp Eye Res. 1996;62:377-387.

33. Akhtar S, Tullo A, Caterson B, Davies JR, Bennett K, Meek KM. Clinical and morphological features including expression of betaig-h3 and keratan sulphate proteoglycans in Maroteaux-Lamy syndrome type B and in normal cornea. Br J Opbthalmol. 2002; 86:147-151

34. Jaiswal JK, Andrews NW, Simon SM. Membrane proximal lysosomes are the major vesicles responsible for calcium-dependent exocytosis in nonsecretory cells. J Cell Biol. 2002;159:625-635.

35. Hoppe W, Rauch U, Kresse H. Degradation of endocytosed dermatan sulphate proteoglycan in human fibroblasts. $\mathrm{J} \mathrm{Biol} \mathrm{Chem.}$ 1988;263:5926-5932.

36. Roughley PG, Mort JS. Catabolism of proteoglycans. In: Iozzo RV, ed. Proteoglycans: Structure, Biology and Molecular Interactions. New York: Marcel Dekker Inc.; 2000;93-111.

37. Kumagai N, Kukuda K, Fujitsu Y, Lu Y, Chikamoto N, Nishida T. Lipopolysaccharide-induced expression of intercellular adhesion molecule- 1 and chemokines in cultured human corneal fibroblasts. Invest Opbthalmol Vis Sci. 2005;46:114-120.

38. Schaefer L, Schaefer RM. Proteoglycans from structural compounds to signalling molecules. Cell Tissue Res. 2010;339:237246.

39. Hayashida Y, Beecher N, Akama TO, et al. Matrix morphogenesis in cornea is mediated by the modification of keratan sulphate by GlcNAc 6-O sulphotransferase. Proc Nat Acad Sci U S A. 2006; 103:13333-13338.

40. Parfitt GJ, Pinali C, Akama TO, et al. Electron tomography reveals multiple self-association of chondroitin sulphate/dermatan sulphate proteoglycans in Chst5-null mouse corneas. J Struct Biol. 2011;174:536-541.

41. Rawe IM, Leonard DW, Meek KM, Zabel RW. X-ray diffraction and transmission electron microscopy of Morquio syndrome type A cornea: a structural analysis. Cornea. 1997;16:369-376.

42. Svensson L, Aszódi A, Reinholt FP, Fässler R, Heinegård D, Oldberg A. Fibromodulin-null mice have abnormal collagen fibrils, tissue organization, and altered lumican deposition in tendon. $\mathrm{J} \mathrm{Biol}$ Chem. 1999;274:9636-9647.

43. Ameye L, Young MF. Mice deficient in small leucine-rich proteoglycans: novel in vivo models for osteoporosis, osteoarthritis, Ehlers-Danlos syndrome, muscular dystrophy, and corneal diseases. Glycobiology. 2002;12:107R-116R.

44. Neame PJ, Kay CJ. Small leucine-rich proteoglycans. In: Iozzo RV, ed. Proteoglycans: Structure, Biology and Molecular Interactions. New York: Marcel Dekker Inc.; 2000;201-235.

45. Alroy J, Haskins M, Birk DE. Altered corneal stromal matrix is associated with mucopolysaccharidosis I, III and VI. Exp Eye Res. 1999;68:523-530.

46. Danielson KG, Baribault H, Holmes DF, Graham H, Kadler KE, Iozzo RV. Targeted disruption of decorin leads to abnormal collagen fibril morphology and skin fragility. J Cell Biol. 1997;136:729743 .

47. Sanches JCT, Jones CJP, Aplin JD, Iozzo RV, Zorn TMT, Oliveira SF. Collagen fibril organization in the pregnant endometrium of decorin-deficient mice. J Anat. 2010;216:144-155.

48. Chakravarti S, Magnuson T, Lass JH, Jepsen KJ, LaMantia C, Carroll H. Lumican regulates collagen fibril assembly: skin fragility and corneal opacity in the absence of lumican. J Cell Biol. 1998;141: 1277-1286. 